\title{
Alveolar Socket Preservation with a Combination of Bone Allografts- A Novel Technique for Bone Preservation and Reconstruction
}

\section{Rishi Bhimani*}

Periodontology, Private Practitioner and Proprietor at Dr. Rishi's Dental Clinic, Navi Mumbai, India

*Corresponding Author: Rishi Bhimani, Periodontology, Private Practitioner and Proprietor at Dr. Rishi's Dental Clinic, Navi Mumbai, India.

Received: July 24, 2019; Published: August 22, 2019

DOI: $10.31080 / A S D S .2019 .03 .0619$

\section{Abstract}

Tooth loss is associated with a variety of etiologies. Whatever may be the reason for tooth extraction, post-extraction healing is unpredictable and commonly results in deformities of the alveolar ridge. Various classic studies have shown that post-extraction resorption process of the alveolus in both jaws is significantly more pronounced on the buccal aspect owing to thinness of bone. Hence, preservation of alveolar socket bone becomes mandatory for future replacement of teeth.

The present case describes a novel technique for socket preservation using a combination of particulate and block bone allografts along with a chorion membrane in a mandibular incisor socket which was extracted due to vertical root fracture. This clinical situation in this case made the employment of classical socket preservation technique impossible. Hence, a novel technique was employed and excellent esthetic as well as functional outcome was achieved.

Keywords: Novel Socket Preservation Technique; Socket Preservation; Ridge Preservation; Bone Grafts; Vertical Root Fracture

\section{Introduction}

Tooth loss is associated with different etiologies like endodontic pathology (root fractures, root perforations); periodontal pathology (combined endodontic-periodontic lesions, periodontal disease); advanced caries lesions; and trauma. Alveolar bone loss can also be iatrogenic, due to traumatic extractions or as a part of natural post-extraction healing process. Whatever the reason for tooth extraction, post-extraction socket healing commonly results in deformities of the alveolar ridge [1].

Because the healing patterns of alveolar bone sockets of humans are unpredictable, tooth extraction may lead to intraoral situations in which the remaining healed ridge does not allow for an esthetic and functional solution without the aid of significant bone grafting. However, it is possible to prevent such problems by simply performing ridge preservation procedures in extraction sockets that employ replacement grafting materials with or without barrier membranes [2].

Various classic studies in the 1960s have shown that the postextraction resorption process of the alveolus in both jaws was significantly more pronounced on the buccal aspect as the buccal surface of the anterior alveolar ridge is commonly thin and fragile.
In a recent prospective study over 1 year follow up period, it was found that the remaining socket/ridge was reduced to $50 \%$ of its original width, greater than $65 \%$ of this reduction occurred within the first 3 months [3]. A comparison of non-molar sockets that healed naturally with similar sockets covered by resorbable membrane found that by 6 months, the first group had an average reduction in alveolar width of $4.56 \mathrm{~mm}$, compared to a reduction of $1.50 \mathrm{~mm}$ in the membrane group [4].

Although in almost any case, the gold standard for bone grafting is the autogenous graft, in socket preservation particularly, it might be aggressive to harvest autogenous bone for such contained defects. Xenografts and allografts have proven reliable for socket preservation as well as they avoid creation of an additional surgical site for harvesting autogenous bone graft [5-7].

Human bone allografts are prepared from cadaveric human donors, processed to eliminate its antigenic properties followed by sterilization. Allografts are available in particulate form or as cortico-cancellous bone blocks of specific sizes. Particle sizes between 250-750 $\mu \mathrm{m}$ have shown to promote osteogenesis [8].

The present case describes the preservation of alveolar socket of a mandibular incisor extracted due to vertical root fracture, 
using a novel combination of Freeze-dried bone allograft (FDBA) particles as well as freeze-dried cortico-cancellous bone block covered by chorion membrane.

\section{Case Report}

A 45-year old male patient reported to Dr. Rishi's Dental Clinic, Navi Mumbai with a chief complaint of pus discharge from the gums of the lower front jaw for 2 weeks. The patient had no relevant medical or drug history.

Intraoral examination revealed a sinus opening with pus discharge along the labial attached gingiva of mandibular right central incisor (41). An isolated deep $10+\mathrm{mm}$ pocket was present labially (Figure 1). There was a history of endodontic therapy with mandibular left (31) and right (41) central incisors 2 years ago. The patient had a deep bite with moderate attrition of mandibular incisors. Radiographic examination revealed a good endodontic seal, but peri-apical bone loss was visible around the apex of 41 . The endodontically treated incisors ( 31 and 41 ) were not crowned.

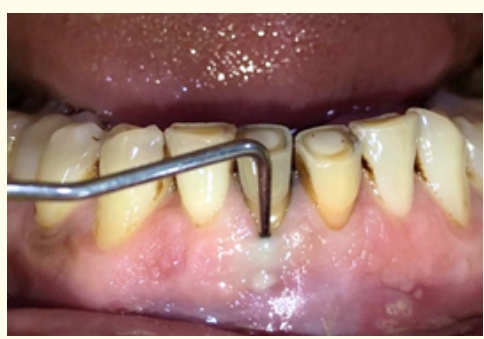

Figure 1: Sinus opening and deep isolated $10+$ mm pocket with 41 .

This clinical condition was suggestive of a root fracture with 41 , although fracture line was not visible radiographically. Surgical exposure of the root was planned after an informed consent from the patient. A full-thickness mucoperiosteal flap was raised with 2-vertical releasing incisions. Vertical root fracture was detected after flap exposure with complete dehiscence of labial cortical bone (Figure 2)]. 41 was carefully extracted (Figure 3). The remaining extraction socket was a 4-walled defect rather than a traditional 5-walled socket defect as the labial bone was completely absent (Figure 4). The socket was curetted and irrigated with saline to remove any debris. FDBA particles (size-500-1000 $\mu \mathrm{m}$ ) procured from TATA TISSUE BANK, TATA MEMORIAL HOSPITAL, PAREL, MUMBAI were mixed with a few drops of saline and placed in the socket defect (Figure 5). As there was no labial cortical bone to secure the graft particles, a cortico-cancellous bone block was used (from the same source) to replace the lost cortex and support graft particles. The $1 \times 1 \times 1 \mathrm{~cm}$ bone block was cut and modified such that a thin sheet of the cortical portion of the cortico-cancellous bone block was used labially, to cover the particulate graft placed in the socket (Figure 6). The bone block was cut conforming to the size and shape of the socket defect. The block was then covered by a chorion membrane (from the same source) (Figure 7). The flap was repositioned, and interrupted sutures were placed with nonresorbable 4-0 braided black silk suture (Figure 8). A periodontal pack (COE-PACK) was placed to protect the surgical site.

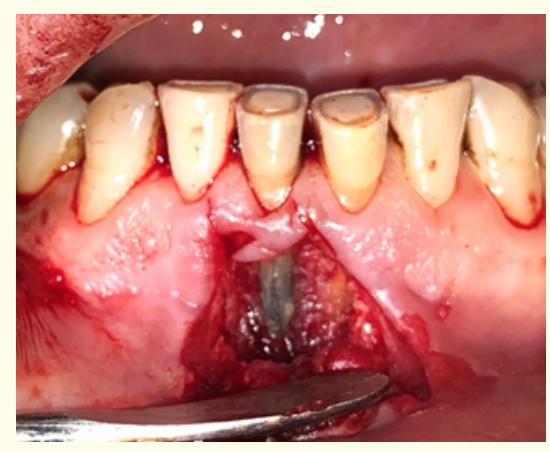

Figure 2: VRF with complete dehiscence of labial cortical bone on flap exposure.

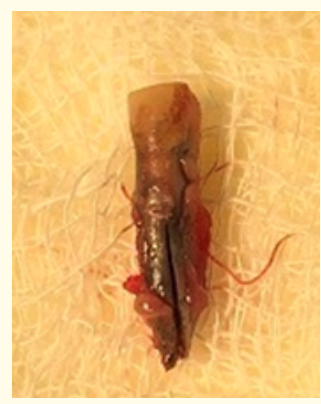

Figure 3: Extracted 41 with vertical root fracture.

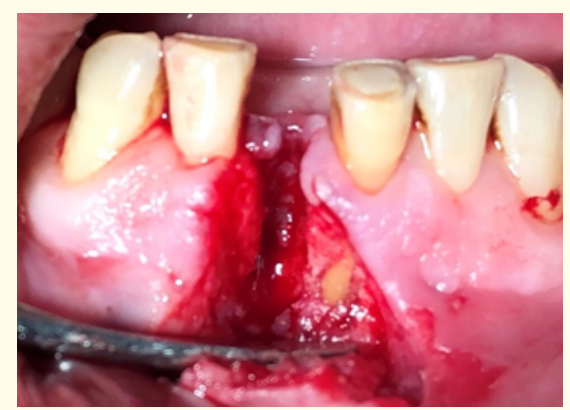

Figure 4: 4-walled socket defect, absent labial cortical bone. 


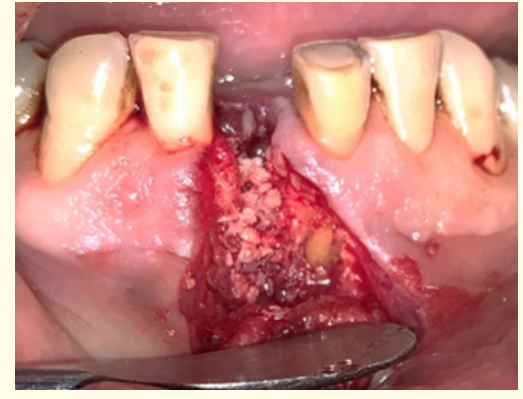

Figure 5: FDBA particulate placed in the socket defect.

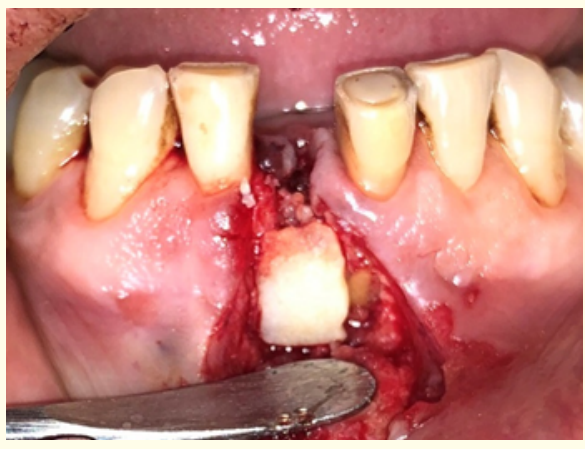

Figure 6: Sheet of cortical portion of cortico-cancellous block placed on the particulate graft.

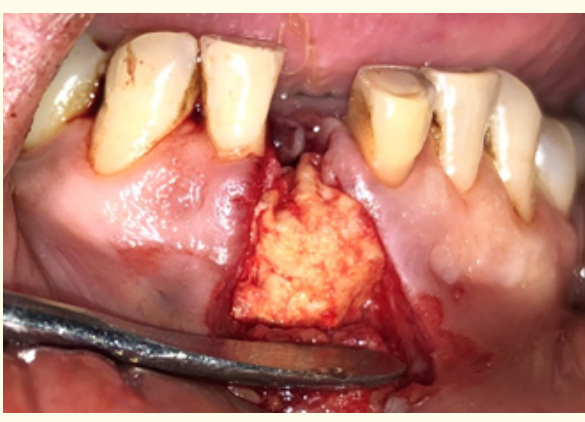

Figure 7: Chorion membrane covering the grafts.

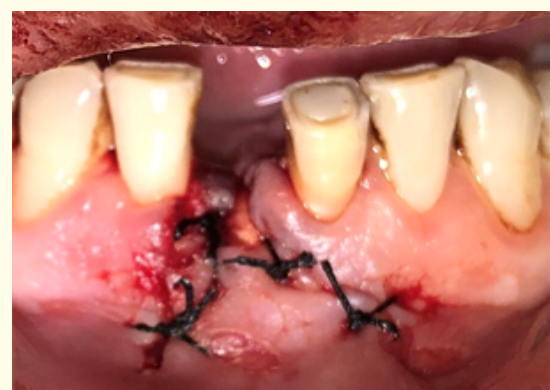

Figure 8: Surgical site sutured.
Post-operative antibiotic- AUGMENTIN 625mg (Amoxycillin $500 \mathrm{mg}+$ Clavulanic acid $125 \mathrm{mg}$ ) twice daily for 5 days and an analgesic-antiinflammatory - DOLONEX DT (Piroxicam $20 \mathrm{mg}$ ) twice daily for 3-5 days were prescribed. Sutures were removed after 1 week. Healing was satisfactory after a week (Figure 9).

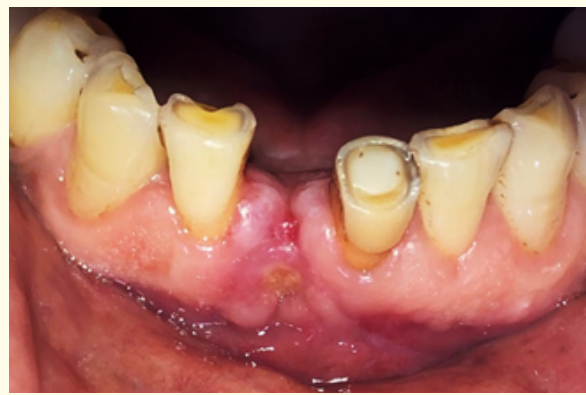

Figure 9: 1-week healing.

Prosthetic rehabilitation was planned for the patient after 4 weeks of complete healing. As the mandibular incisors were severely attrited, endodontic therapy was performed with 32 and 42, after which a 4-unit (metal-free) ceramic fixed partial denture was fabricated and fixed from 32-42 (Figure 10). Excellent esthetics was achieved as well as a pleasing soft tissue contour was maintained at the grafted site. The patient has been maintained on a regular 6-month follow up.

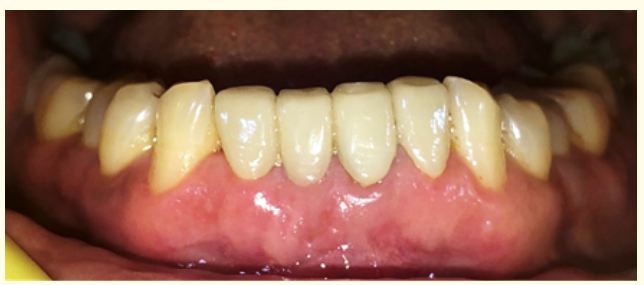

Figure 10: Final healing and outcome after tooth replacement at 6 weeks.

\section{Discussion}

A "true" vertical root fracture (VRF) is defined as a complete or incomplete fracture initiated from the root at any level, usually directed bucco-lingually" [9]. VRF poses diagnostic challenges. The present case was a true VRF associated with complete loss of labial cortical bone and a deep isolated pocket, up to the root apex.

Classical socket preservation techniques employ placement of a particulate graft material into the socket, followed by covering with a membrane. Then, the flap is sutured back with coronal advancement. This technique can be carried out if the socket defect is well contained. 
In the present case, a thin sheet of cortical bone was cut from the cortical side of the cortico-cancellous bone block, conforming to the shape and size of the defect, placed over the lost labial cortex. The purpose was to contain the socket defect filled with FDBA particles and provide strong mechanical support. Additionally, the incorporation of a cortical allograft is preceded by creeping substitution, which is similar to its autogenous counterpart. In general, the process is initiated by the osteoclastic resorption and followed by sporadic formation of new appositional bone through osteoconduction and osteointegration [10]. The particulate grafts serve well as fillers in contained defects and get incorporated slowly as compared to autografts. Hence, a combination of bone grafts was used for alveolar reconstruction in the present case.

A chorion membrane was used as a barrier, to cover the bone grafts labially and coronally. Chorion membrane has numerous advantages owing to its structure and composition. Moreover, the membrane is dry and quickly hydrates with blood, becomes very pliable, and closely adapts to the contours of the underlying alveolar bone. It is self-adherent in nature and does not need to be fixed into place using sutures. It is resorbable, avoiding secondary surgical intervention for its removal. It contains collagen Types I to VII, proteoglycans, fibronectin and laminins. Immunohistochemical staining analysis showed intense concentrations of laminin and laminin-5 throughout the barrier which is of importance due to its high affinity for binding gingival epithelial cells for better adaptation to the root surface [11].

Excellent soft tissue healing was achieved at 15 days after the grafting procedure. The gingival contour was maintained labiolingually as well as apico-coronally in accordance with the soft tissues of adjacent teeth.

In this case, the patient's esthetic and functional demands were met satisfactorily with a very good treatment outcome.

\section{Conclusion}

Clinical scenarios like these are frequently encountered during dental treatment where the thin labial cortical bone resorbs postextraction or is previously missing (as in this case) due to trauma or chronic infection, especially in the maxillary and mandibular anterior regions. Such sequelae can be detrimental for tooth replacement. Hence, it is important for every dental practitioner to predict the post-extraction healing outcome to prevent further bone resorption and attempt to reconstruct and maintain bone through socket preservation techniques.

\section{Bibliography}

1. Mecall RA and Rosenfeld AL. "Influence of residual ridge resorption patterns on implant fixture placement and tooth position 1". International Journal of Periodontics \& Restorative Dentistry 11 (1991): 8-23.

2. Zubillaga G., et al. "Changes in alveolar bone height and width following post-extraction ridge augmentation using a fixed bioabsorbable membrane and demineralized freeze-dried bone osteoinductive graft". Journal of Periodontology 74 (2003): 965-975.

3. Schropp L., et al. "Bone healing and soft tissue contour changes following single-tooth extraction: a clinical and radiographic 12-month prospective study". International Journal of Periodontics \& Restorative Dentistry 23 (2003): 313-323.

4. Lekovic V., et al. "Preservation of alveolar bone in extractions sockets using bioabsorbable membranes". Journal of Periodontology 69 (1998): 1044-1049.

5. Iasella JM., et al. "Ridge preservation with freeze-dried bone allograft and a collagen membrane compared to extraction alone for implant site development: a clinical and histologic study in humans". Journal of Periodontology 74 (2003): 990-999.

6. Artzi Z., et al. "Porous bovine bone mineral in healing of human extraction sockets. Part 1: histomorphometric evaluations at 9 months". Journal of Periodontology 71 (2000): 1015-1023.

7. Carmagnola D., et al. "Healing of human extraction sockets filled with Bio-Oss". Clinical Oral Implants Research 14 (2003): 137-143.

8. Grover V., et al. "Bone allografts: A review of safety and efficacy". Indian Journal of Dental Research 22.3 (2011): 496-500.

9. American Association of Endodontics. Endodontics: Colleagues for Excellence- Cracking the cracked tooth code: Detection and Treatment of Various Longitudinal Tooth Fractures. (2008): 1-8.

10. Bhatt R A and Rozenthal TD. "Bone graft substitutes". Hand Clinics 28 (2012): 457-468.

11. Bryant-Greenwood GD. "The extracellular matrix of the human fetal membranes: Structure and function". Placenta 19 (1998): 1.

\section{Volume 3 Issue 9 September 2019 (C) All rights are reserved by Rishi Bhimani.}

